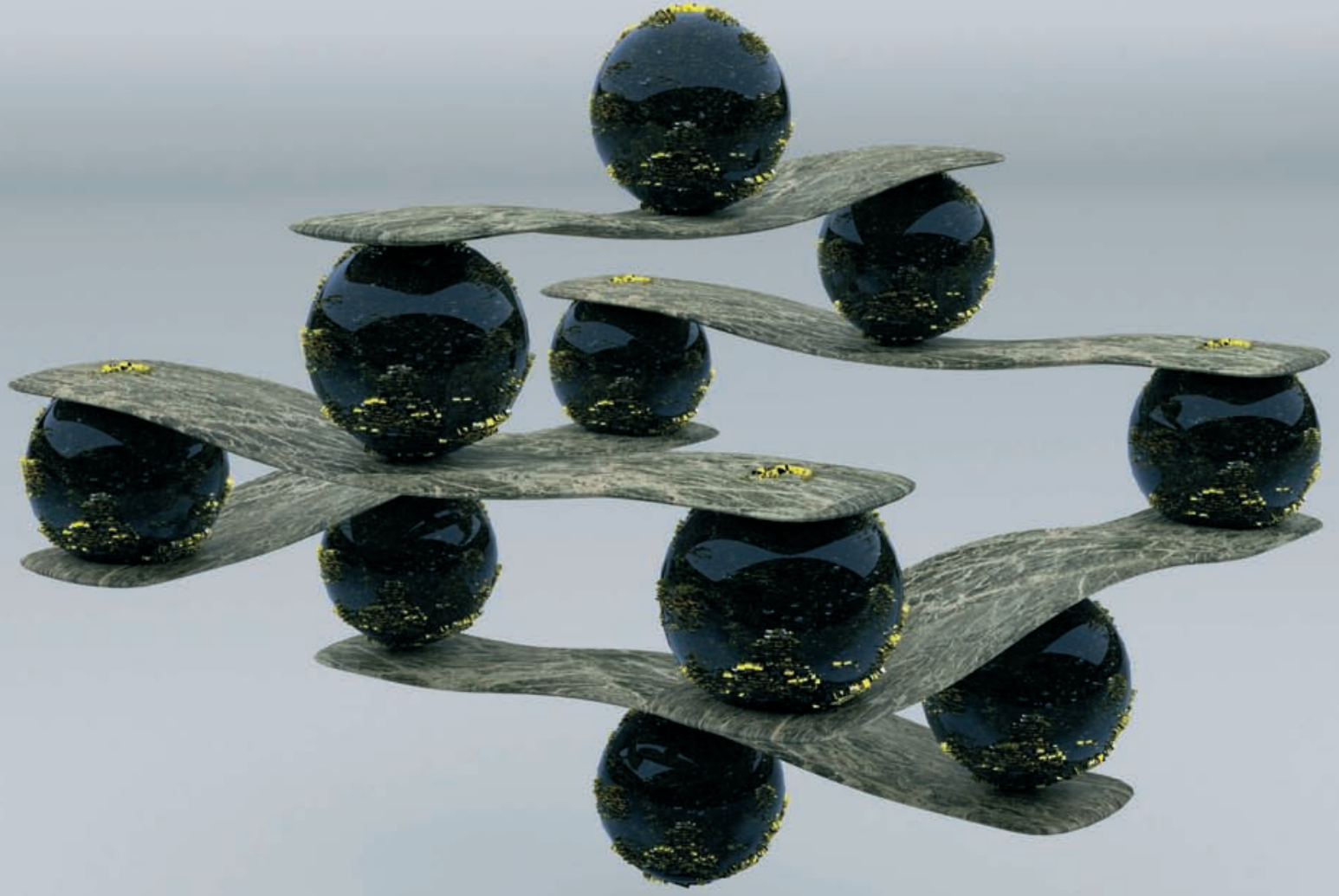

\title{
Zurück ins
}

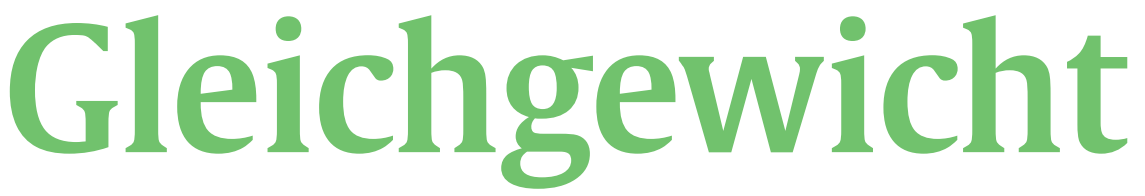

\section{Die ÖSTRADIOLDOMINANZ ist eine häufig vorkommende Form der hormonellen Dysbalance - und oft hausgemacht!}

Marianne Krug

MIGRÄNE, INFERTILITÄT, ERSCHÖPFUNG. Es ließe sich eine lange Liste fortschreiben mit Funktionsstörungen und Erkrankungen, deren Ursachen mit Begriffen wie hormonelle Dysbalance bzw. hormonelles Ungleichgewicht überschrieben sind. Immer öfter tauchen beide Begriffe mittlerweile in den Fach- und Publikumsmedien auf. Auch bei Fachkongressen ist die hormonelle Dysbalance oft Thema.

In den letzten Jahren ist es v.a. eine Dysbalance im Hormonsystem, die bei Frauen immer häufiger festgestellt wird, mitunter aber auch bei Männern: die Östradioldominanz. Längerfristig kann sie bei Frauen zu Uterusmyomen, Zervixdysplasie, Endometriumkarzinomen, Eierstockzysten und -karzinomen sowie Brustkrebs führen (weitere Erkrankungen und Beschwerdebilder, s. S.37). Bei Männern steht sie in Verdacht, Prostatakarzinome zu verursachen.
Bei Frauen kann es während des Klimakteriums zeitweise physiologisch zu einer Östradioldominanz kommen. Doch es gibt auch andere Ursachen, die dazu führen können und die nicht als physiologisch zu betrachten sind.

\section{Aus dem Gleichgewicht geraten}

Unser Hormonsystem ist hierarchisch organisiert. Es gibt Steuerungshormone und Rückkopplungsmechanismen. Hormone können auf andere fördernd, ebenso aber auch antagonisierend wirken. Diese Wechselbeziehungen der Hormone untereinander lassen sich sehr gut mit einem Mobile vergleichen: Ein Mobile hat verschiedene Etagen, wobei jede Etage über mindestens 2 einander balancierende Enden verfügt. Damit das Mobile stabil ist, müssen alle Etagen in Balance sein. Ganz ähnlich verhält es sich 
mit den Hormonen: Sie müssen in einem ausgewogenen Verhältnis vorhanden sein. Ist von einem zu viel oder zu wenig vorhanden, stört das die Balance und beeinflusst das gesamte System. Bei einer Östradioldominanz sind es die Hormone Östradiol, Testosteron und Progesteron, die nicht mehr in Balance sind.

\section{„Weibliche" und „männliche“" Hormone?}

Östradiol und Testosteron zählen zu den Steroidhormonen und innerhalb dieser Gruppe zu den Sexualhormonen. Östradiol wird landläufig als „weibliches“, Testosteron als „männliches“ Hormon bezeichnet. Das hat eigentlich keinen Sinn, denn sowohl Männer als auch Frauen bilden Testosteron und Östradiol aus, z.T. schon ab der 6. Fetalwoche. Im Körper von Frauen und Männern ist der Gehalt des Hormons Östradiol (im englischen Sprachraum als Estradiol bezeichnet, es ist eines von derzeit 3 bekannten Östrogenen) und des Hormons Testosteron physiologisch zwar unterschiedlich hoch, im Normalfall ist das Verhältnis zueinander jedoch stets fein ausbalanciert.

Östradiol und Testosteron haben eine kraftspendende, energetisierende, vitalisierende, gewebeaufbauende und zellwachstumsfördernde Wirkung (weitere Wirkungen, s.S. 50).

Das Progesteron (Gelbkörperhormon), ebenfalls ein Steroidhormon aus der Gruppe der Sexualhormone, ist der notwendige Gegenpol zu Testosteron und Östradiol. Es hat eine beruhigende, schlaffördernde, angstlösende, entspannende, entkrampfende, entwässernde und abschwellende Wirkung (weitere Wirkungen, s.S. 51).

\section{Ursache des Ungleichgewichts: Häufig ist es der Lebensstil}

Die Ursachen für eine Dysbalance von Östradiol, Testosteron und Progesteron, die zu einer Östradioldominanz führen können, vom

\section{Erkrankungen und Beschwerde- bilder, die auf eine Östradiol- dominanz zurückzuführen sind}

\footnotetext{
- Dysmenorrhö/Hypermenorrhö

- Zyklusstörungen

- zyklusabhängige depressive Verstimmungen und Stimmungsschwankungen

- Prämenstruelles Syndrom (PMS)

- Infertilität/Subfertilität

- Mastopathie

- Schlafstörungen

- hormonell bedingte Migräne

- Leistungsminderung

- alle prämenopausalen und postmenopausalen Beschwerden

- Malignome der hormonabhängigen Gewebe

- Nicht zu vergessen: Erschöpfungssyndrome durch stressbedingten Kortisolüberschuss
}

\section{KURZ GEFASST \\ 1 Adipositas, vermehrter Alkohol- und Süßigkeitenkonsum, Dauer- \\ stress, aber auch Xenoöstrogene in Wasser und Lebensmitteln sind bedeutsam für die Entstehung der Östradioldominanz. \\ 2 Die Diagnose erfolgt über die Hormonspiegelbestimmung im Speichel. Bei der Interpretation der Ergebnisse sind nicht nur die absoluten Werte zu betrachten, sondern auch die Mengenver- hältnisse der Hormone zueinander. \\ 3 Neben der Reduktion der Noxen zur Senkung bei erhöhtem Östradiolgehalt kann die Substitution von DHEA, Testosteron und Progesteron bei Mangel indiziert sein.}

Klimakterium abgesehen, scheinen auf den ersten Blick unterschiedlich. Sieht man aber genauer hin, erkennt man eine Gemeinsamkeit: Sie sind häufig unserem Lebensstil bzw. unserem Lebensstilwandel in den letzten Jahrzehnten geschuldet.

\section{Östradiol- und Testosteron-Dysbalance}

Östradiol und Testosteron werden größtenteils in den Gonaden (Keimdrüsen) gebildet. Mithilfe des Enzyms Aromatase können jedoch sowohl Männer als auch Frauen Testosteron zudem in ihren Fettzellen zu Östradiol umwandeln. Je größer also der Körperfettanteil, umso ausgeprägter die Östradiolsynthese und umso höher der Östradiol- bzw. geringer der Testosterongehalt im Körper. Das erklärt, warum gerade bei Adipösen, deren Anzahl in den letzten Jahren weltweit drastisch gestiegen ist, häufig Symptome auftreten und Erkrankungen und Beschwerdebilder diagnostiziert werden, die durch eine Östradioldominanz verursacht werden.

Die gesteigerte Östradiolsynthese durch Aromatase kann jedoch noch andere lebensstilbedingte Ursachen haben: Alkohol und zuckerreiche Nahrungsmittel führen zu einer Erhöhung des Insulinspiegels mit Stimulation der Aromatase-Aktivität. Die Zunahme der Östradioldominanz kann somit auch auf den in den letzten Jahrzehnten in den Industrienationen massiv gesteigerten Alkohol- und Süßigkeitenkonsum zurückgeführt werden.

Stress kann eine weitere Ursache für eine Östradioldominanz gegenüber Testosteron sein. Dauerstress führt zu einer Leistungsminderung der Nebennieren, wodurch es früher oder später zu einem Absinken der Gesamtandrogene kommt, zu denen Testosteron zählt. Diese Gruppe der Sexualhormone hat auch bei Frauen Einfluss auf die muskuläre Leistungsfähigkeit, Ausdauer, Stresstoleranz und nicht zuletzt die Libido. Die Vorstufe des Testosterons, das sog. DHEA (Dehydroepiandrosteron) wird bei Männern zu 50\% und bei der Frau zu 90\% in den Nebennieren gebildet.

Merke: Bei Frauen mit hormonell bedingten Erkrankungen bzw. Beschwerden wird bisher kaum an einen Mangel oder Überschuss an Androgenen gedacht. 


\section{0\% aller Erstverschreibungen der Pille nehmen}

\section{derzeit Dermatologen vor. Sie verordnen das}

\section{Hormonpräparat gegen Akne und Hirsutismus.}

\section{Progesteronmangel: Schuld ist häufig die Pille}

Ein Grund, warum in den vergangenen 50 Jahren die Zahl der Frauen drastisch gestiegen ist, bei denen die fein ausgeglichene Balance der Hormone Östradiol und Progesteron ins Wanken gerät, ist die Ovulationshemmung. Frauen synthetisieren Progesteron größtenteils im Gelbkörper, der sich nach dem Eisprung aus der verbleibenden Follikelhülle bildet. Eine Follikelhülle kann jedoch nur nach erfolgter Ovulation entstehen. Eine Verhinderung des Eisprungs führt daher zu einer fast $60 \%$ igen Abnahme des Progesterongehalts im Körper der Frau. Im Speicheltest von Frauen, die Ovulationshemmer einnehmen, ist daher oft ein um 40$50 \%$ verminderter Progesteronspiegel nachweisbar.

Doch nicht nur Pille und Hormonspirale führen zum Absinken des Progesteronspiegels. Stress ist eine weitere Ursache. Denn Progesteron ist eine Hormonvorstufe anderer Steroide, u.a. des Kortisols. Bei erhöhter Stressbelastung kommt es daher zu einer Art „Steel-Phänomen“: Der Körper bedient sich des Progesterons, um bei Stress genügend Kortisol synthetisieren zu können und den Kortisolspiegel für die Stressbewältigung entsprechend zu erhöhen.

Die inadäquate Zufuhr des Hormons als Teil der konventionellen Hormonersatztherapie (HET, s.S. 48-51) kann ebenfalls zur Östradioldominanz führen.

\section{Simulierte Östradioldominanz}

Nicht zu vergessen: Die Wirkung der Xenoöstrogene auf den Körper. Xenoöstrogene sind synthetisch hergestellte chemische Verbindungen mit östradiolartiger Wirkung. Dazu zählen u.a. verschiedene Pestizide, Bisphenole sowie einige Phthalate, die als Verunreinigung in der Umwelt und in Lebensmitteln auftreten können, z. B. DDT, Endosulfan, Bisphenol A, Methoxychlor, 4-Hydroxy-2',4',6'-trichlorbiphenyl, 4-tert-Octylphenol, 4-Nonylphenol, Butylbenzylphthalat und D-n-Butylphthalat. Persistente Stoffe wie Organochlorpestizide und polychlorierte Biphenyle können sich in der Nahrungskette anreichern und finden sich in Spuren praktisch überall. Besonders belastet sind Lebensmittelverpackungen oder Plastikflaschen.

Die östradiolartige Wirkung der Xenoöstrogene basiert darauf, dass sie an Östradiolrezeptoren andocken. Sind sie in einer entsprechend hohen Konzentration im Körper vorhanden, simulieren sie eine Östradioldominanz und führen zu denselben Symptomen und Krankheitsbildern.

\section{Auch Männer bilden Progesteron}

Progesteron wird zwar hauptsächlich, aber nicht ausschließlich in der Follikelhülle gebildet, sondern auch in der Nebennierenrinde. Das erklärt, warum auch im Körper von Männern Progesteron vorkommt.

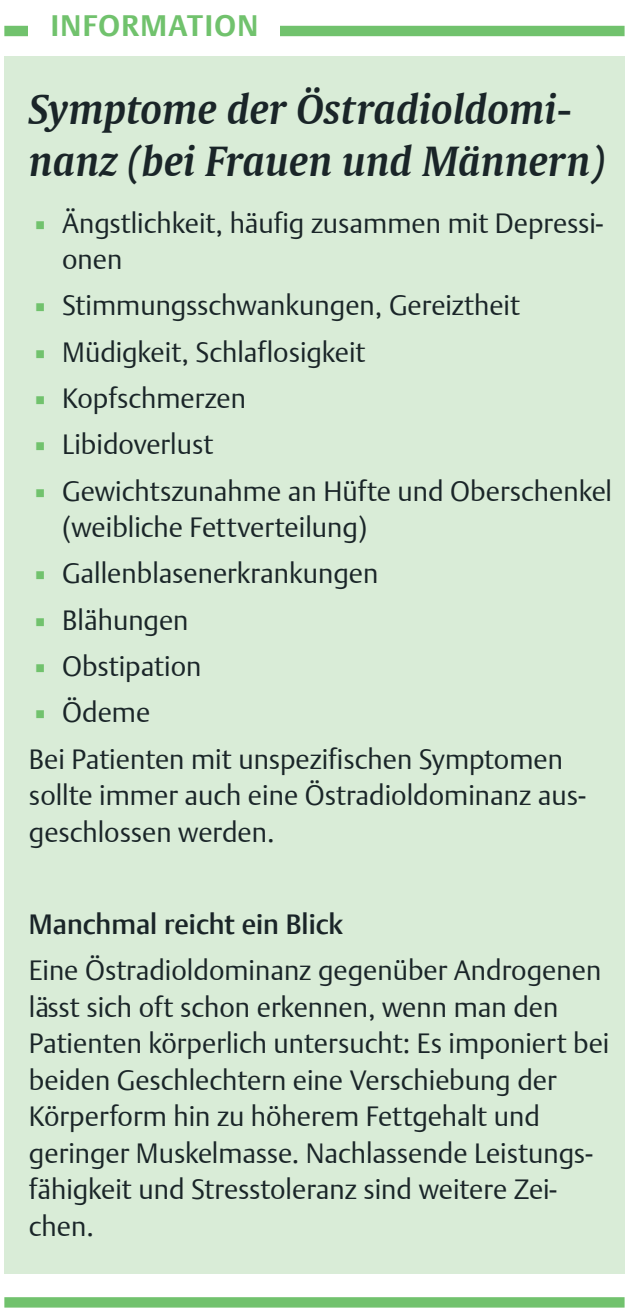

\section{Diagnostik: Hormone im Mengenverhältnis zueinander betrachten}

Für die Diagnostik einer Östradioldysbalance ist es wichtig, die Menge der Steroidhormone Östradiol, Testosteron, Progesteron und DHEA zu bestimmen, die den Zellen zur Verfügung steht und an Rezeptoren binden kann. 95\% der Hormone im Serum sind an Transporteiweiße gebunden, z. B. das SHBG (Sexualhormonbindendes Globulin) oder Albumine, und somit metabolisch inaktiv. Das bedeutet: Die gemessenen Serumspiegel geben zwar einen Überblick über die Gesamtmenge der einzelnen Hormone im Körper - nicht aber über die tatsächliche Verfügbarkeit. Deshalb setzt man bei der Diagnostik der hormonellen Dysbalance bevorzugt den Speicheltest ein (s.S. 18-23).

Bei der Betrachtung der Ergebnisse des Speicheltests achtet man bei den Hormonen Östradiol, Testosteron, Progesteron und DHEA nicht nur auf die absoluten Werte. Die Mengenverhältnisse der Hormone zueinander, auch und gerade innerhalb angegebener Normwerte, sind wichtig, um beurteilen zu können, ob noch eine Balance besteht oder bereits eine Dysbalance vorliegt, und wie stark diese ausgeprägt ist.

Beispielsweise wird als eine Bezugsgröße das Verhältnis von Östradiol zu Progesteron angegeben. Dies ist bei einer Frau in der 
Verhältnis Östradiol zu Testosteron

Frauen (prämenopausal): 1:6

Frauen (postmenopausal): 1:10

Männer: 1:10

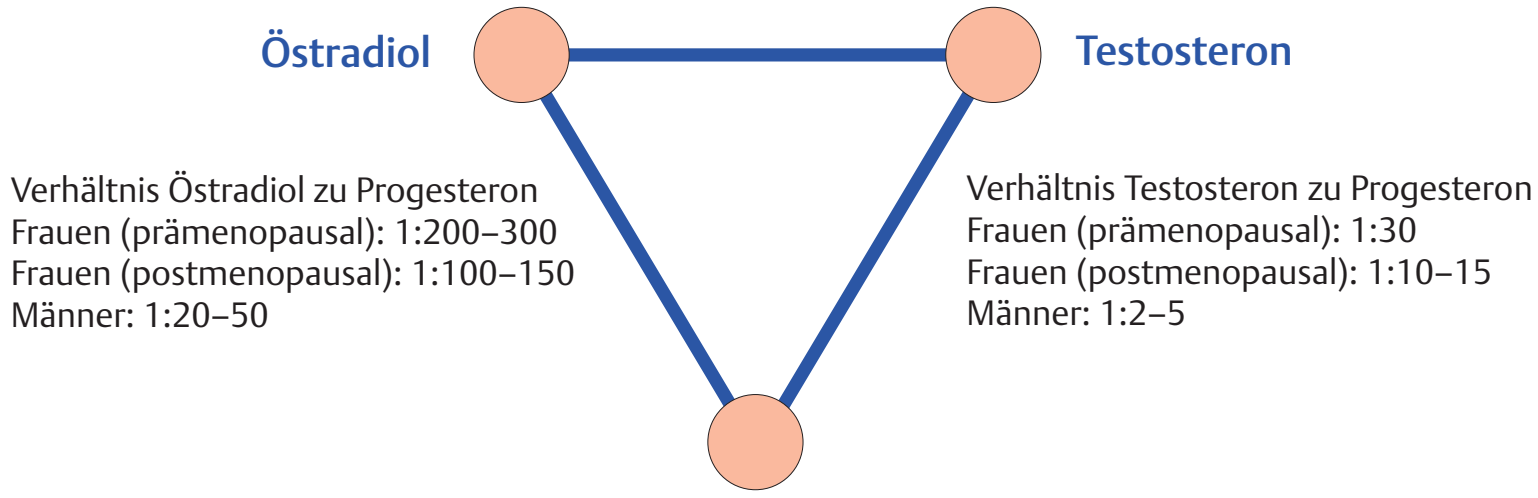

Progesteron

fertilen Phase im Idealfall 1:200, postmenopausal ist auch ein Verhältnis von 1:100 ausreichend. Bei Männern geht man von 1:50 als idealem Verhältnis aus.

\section{Absolute Östradioldominanz:}

\section{Lebensstiländerung ist das A \& 0}

Bei einer absoluten Erhöhung des Östradiolgehalts im Körper (Werte liegen oberhalb der vom Labor angegebenen Normwerte) hat dessen Senkung Vorrang vor allen anderen Maßnahmen. Diese beginnt bei Adipösen und Patienten mit einem zu hohen Körperfettanteil mit der Reduktion des Fettgewebes. Hinzu kommen Lebensstiländerungen wie Reduktion des Alkoholkonsums, Zuckerrestriktion und Reduktion schnellresorbierbarer Kohlenhydrate, um dadurch die Aromatase-Aktivität zu senken. Ganz wichtig ist es, auch die Xenoöstrogene zu reduzieren.

Eine absolute Erhöhung des Östradiolgehalts geht immer auch mit einer proliferativen Wirkung (das Zellwachstum fördernd) einher. Das bedeutet: Das statistische Risiko einer malignen Entartung steigt. Es gilt daher, die hormonabhängigen Gewebe vor dem Östradiol und einigen risikoreichen Metaboliten, z. B. 16-OH-Östron und 4-OH-Östron, zu schützen.

Bewährt hat sich dazu die Gabe von Isoflavonen aus Soja oder Rotklee zusammen mit einem Phytopharmakon, das Indol-3-Carbinol enthält. Isoflavone besetzen die ß-Östrogen-Rezeptoren und lösen dort eine schwache Östradiolwirkung aus - im Gegensatz zu der starken Östradiolwirkung des körpereigenen oder zugeführten 17ß-Östra- diol. Es gibt 2 unterschiedliche Östrogen-Rezeptoren: $\alpha$-ÖstrogenRezeptoren bewirken u.a. eine Proliferation des Endometriums und der Mammae. ß-Östrogen-Rezeptoren wirken antiproliferativ und hemmen gleichzeitig die Aktivität der $\alpha$-ÖstrogenRezeptoren. Indol-3-Carbinol kann die Metabolisierung des Östradiols in risikoarme Metabolite bewirken.

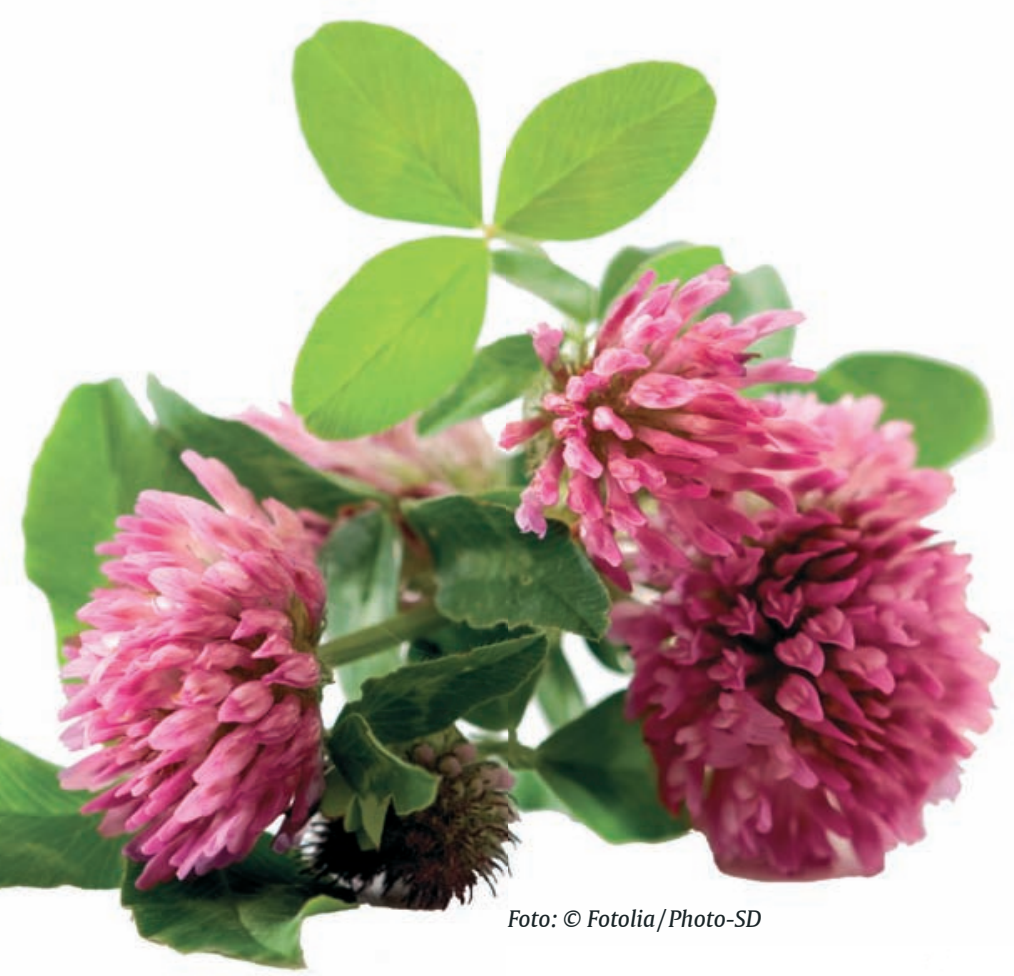




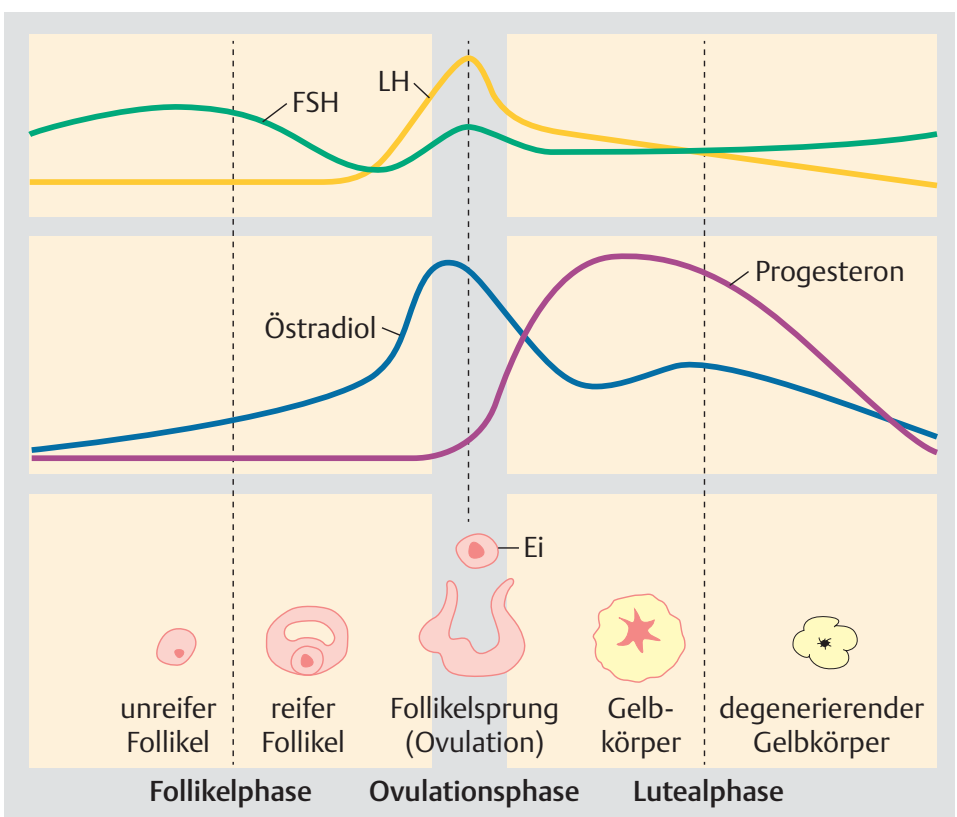

Isoflavonhaltige Präparate gibt es in Deutschland von vielen Herstellern, z. B. femiloges ${ }^{\circledR}$ (Fa. Loges / Dosierung: $2 \times \operatorname{tgl}$. 2 Kps.), Rotklee Plus (Fa. Fairvital BV/Dosierung: 1 tgl. 1 Kps.), Biogena IMK $^{\circledR}$ Isoflavone (Fa. Biogena IMK). Indol-3 Carbinol ist als Reinsubstanz z. B. als Indol-3 Carbinol (Fa. Fairvital/Dosierung: 2-4 Kps. tgl.) oder als I3C ${ }^{\circledR}$ (Fa. Vitabasix/Dosierung: 2 Kps. tgl.) erhältlich. Ein Kombinationspräparat, das Isoflavone und Indol3-Carbinol in ausreichender Wirkstoffkonzentration enthält, ist derzeit nur in den USA erhältlich.

\section{Relative Östradioldominanz}

Eine relative Östradioldominanz (Werte liegen noch innerhalb angegebener Normwerte im oberen Bereich) lässt sich durch eine Erhöhung der Hormone, die im Verhältnis in zu geringen Mengen vorhanden sind, beheben.

Ist der Organismus des Patienten regulationsfähig, lassen sich z.B. mit Mönchspfeffer die Progesteronproduktion bei Männern sowie Frauen und mit Erd-Burzeldorn (Tribulus terrestris) oder Chinesischem Raupenpilz (Cordiceps sinensis) die Produktion der Androgene stimulieren. Beispiele für geeignete Präparate:

- Mönchspfeffer (Vitex agnus-castus), z. B. Agnolyt ${ }^{\circledR}$

(Fa. Madaus), Dosierung: morgens $1 \mathrm{Kps}$.

- Erd-Burzeldorn (Tribulus terrestris), z. B. Tribulus

(Fa. Vitabasix), Dosierung: 1-3 Kps. tgl.

- Chinesischer Raupenpilz (Cordyceps sinensis),

z. B. Cordyceps Extrakt (Fa. Fairvital), Dosierung: 3 Kps. tgl.

Ist die Regulationsfähigkeit dagegen eingeschränkt oder nicht mehr vorhanden (z.B. bei Ovarialinsuffizienz, Lutealinsuffizienz oder auch Hypogonadismus), ist eine Hormonsubstitution angezeigt, also der Ersatz humanidentischer bzw. bioidentischer Hormone.

Bioidentische Steroidhormone werden zumeist aus einer pflanzlichen Vorstufe aus der wilden Yamswurzel (Dioscorea villosa, s.S. 44-46) synthetisiert. Fälschlicherweise werden häufig Extrakte der Yamswurzel eingesetzt unter der Vorstellung, damit zu substituieren. Der menschliche Körper besitzt allerdings nicht die Enzyme, die notwendig sind, um die Hormonvorstufe aus der Yamswurzel entsprechend zu synthetisieren. Es braucht daher eine biochemische Aufbereitung.

Die bioidentischen Hormone Progesteron oder Testosteron können sehr einfach als Cremezubereitung transdermal zugeführt werden. Bei richtiger Gelgrundlage lassen sich mit geringen Dosen hohe Gewebsspiegel erzeugen. Es handelt sich dabei über-
Neulich suchte mich die 50 -jährige Elke $\mathrm{H}$. in meiner Praxis wegen eines „Hormonthemas“ auf. Sie sei nie krank gewesen und habe nie irgendwelche Beschwerden gehabt, so die Patientin. Vor etwa einem Jahr sei dann plötzlich ihre Menstruation ausgeblieben. Kurz zuvor habe sie öfter Herzstolpern bemerkt, Hitzewallungen seien aufgetreten, zusammen mit Durchschlafstörungen. Zudem habe sie seit dem Ausbleiben der Mensis kaum noch Lust auf oder Spaß am Sex. Stimmungsschwankungen seien ihr und ihrer Umgebung aufgefallen, sie könne sich deutlich schlechter konzentrieren als früher, und zudem sei sie auch nicht mehr so leistungsfähig.

Ihre Gynäkologin habe ihr ein Kombinationspräparat aus Östradiol und Gestagen verschrieben. Zwar verschwanden die Hitzewallungen kurz nachdem sie mit der Einnahme begonnen habe. Unter gelegentlichem Herzstolpern, Stimmungsschwankungen und Konzentrationsstörungen habe sie jedoch weiterhin sehr zu leiden.

Ein Speicheltest ergab eine Östradiolkonzentration im Normbereich - das ist erklärlich, da in dem verordneten Kombinationsprä- parat bioidentisches Östradiol enthalten ist. Es zeigte sich aber auch ein deutliches Missverhältnis von Östradiol zu Testosteron und Progesteron: Das Verhältnis Östradiol zu Testosteron lag bei $1: 2$ (statt $1: 10$ ), das Verhältnis Östradiol zu Progesteron bei $1: 9$ (statt 1:100). Trotz der Östradiolwerte im Normbereich zeigte sich bei der postmenopausalen Patientin also ein relativer Androgen- und relativer Progesteronmangel.

Dies ist ein Effekt, der regelmäßig unter der Gabe eines Gestagenpräparats auftritt, denn es supprimiert die Androgene und das körpereigene Progesteron.

Meine Therapie bestand darin, das Kombinationspräparat Zug um Zug durch bioidentisches Östradiol, Progesteron und Testosteron zu ersetzen. Drei Monate später waren die Werte im Speicheltest perfekt angestiegen: Das Verhältnis Östradiol zu Testosteron lag mit $1: 8,5$ fast im Normwertebereich. Das Verhältnis Östradiol zu Progesteron lag bei 1:200. Die Dosierung des Progesterongels konnte in der Folge reduziert werden. Die ersten Worte der Patientin beim Kontrollbesuch waren: „Es ging mir schon lange nicht mehr so gut!“ 
wiegend um verschreibungspflichtige Präparate. Heilpraktiker dürfen lediglich Cremezubereitungen in der nicht verschreibungspflichtigen Potenz D4 einsetzten.

Merke: Bioidentische Hormone dürfen nur nach kritischer Risiko-Nutzen-Beurteilung und ggf. Rücksprache mit dem behandelnden Gynäkologen oder Allgemeinmediziner eingesetzt werden. Eine sorgfältige Dosierung unter regel-

mäßiger Hormonspiegelkontrolle ist Voraussetzung!

Der Körper erhält durch diese Substitutionstherapie ähnlich wie in der orthomolekularen Behandlung das, was er aus eigener Kraft vorübergehend oder dauerhaft nicht in ausreichendem Maße zur Verfügung stellen kann.

Dieser Artikel ist online zu finden:

http://dx.doi.org/10.1055/s-0035-1569238

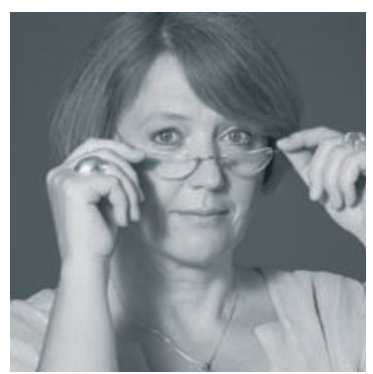

\section{Marianne Krug}

Große Friedberger Str. 44-46

60313 Frankfurt am Main

E-Mail: kontakt@mariannekrug.de

Marianne Krug ist Ärztin. Seit 1982 ist sie als Fachärztin für Allgemeinmedizin in eigener Praxis niedergelassen mit den Schwerpunkten Akupunktur, Mikrobiologische Therapie, Naturheilverfahren, hämatogene Oxydationstherapie. 1999 hat sie sich zudem auf die natürliche Hormonersatztherapie, Schilddrüsenbehandlung, Präventivmedizin und Anti-Aging-Medizin spezialisiert. Seit 2001 Vortrags- und Seminartätigkeit zu diesen Spezialgebieten. 